\title{
Commentary \\ A 10-Year Perspective on the Utility of Three Adjuvants Often Used in IVF: Growth Hormone, Melatonin and DHEA
}

\author{
John L. Yovich ${ }^{1,2, * \mathbb{C}}$ and Peter M. Hinchliffe ${ }^{1}$ \\ 1 PIVET Medical Centre, Perth, WA 6007, Australia; apwin@pivet.com.au \\ 2 Department of Pharmacy and Biomedical Sciences, Curtin University, Perth, WA 6845, Australia \\ * Correspondence: jlyovich@pivet.com.au
}

check for updates

Citation: Yovich, J.L.; Hinchliffe, P.M. A 10-Year Perspective on the Utility of Three Adjuvants Often Used in IVF: Growth Hormone, Melatonin and DHEA. Reprod. Med. 2021, 2 , 155-162. https://doi.org/10.3390/ reprodmed 2040016

Academic Editor: Berthold Huppertz

Received: 2 October 2021

Accepted: 3 November 2021

Published: 4 November 2021

Publisher's Note: MDPI stays neutral with regard to jurisdictional claims in published maps and institutional affiliations.

Copyright: (C) 2021 by the authors. Licensee MDPI, Basel, Switzerland. This article is an open access article distributed under the terms and conditions of the Creative Commons Attribution (CC BY) license (https:// creativecommons.org/licenses/by/ $4.0 /)$.

\begin{abstract}
Since 2010, numerous studies reported from PIVET, a pioneer IVF facility established over 40 years ago, have explored the use of three adjuvants designed to improve laboratory and clinical outcomes in cases where a poor prognosis has been demonstrated. The adjuvants reported commenced with recombinant growth hormone $(\mathrm{rGH})$, followed by dehydroepiandrosterone (DHEA) after developing a unique troche to avoid the first-pass effect and, subsequently, melatonin. The studies show that rGH is beneficial in the situation where women have poor-quality embryos in the setting of additional poor prognosis factors, such as advanced female age, a very low ovarian reserve, an insulin growth factor profile in the lowest quartile or recurrent implantation failure. The studies also imply that the adjuvants may actually reduce live birth productivity rates if used on women without poor prognosis factors; hence, further studies, which can now be better designed, should be undertaken to explore the notion of underlying adult growth hormone deficiency in some cases as well as the suggestion that DHEA can provide equivalent benefits in some poor prognosis settings. Melatonin showed no suggestive benefits in any of the studies and can be excluded from consideration in this context. Future studies should compare rGH and DHEA with a focus on those women who have poor embryo quality with additional poor prognosis factors. Such trials should be extended to 12 weeks to cover the entire period of oocyte activation.
\end{abstract}

Keywords: in vitro fertilisation (IVF); intracytoplasmic sperm injection (ICSI); adjuvants; recombinant growth hormone (rGH); melatonin; dehydroepiandrosterone (DHEA); live birth productivity rate (LBPR)

\section{Introduction}

Although assisted reproductive technology (ART) has earnt a well-respected position in modern medicine with its current widespread application generating more than 10 million offspring since the first child born in 1978, in truth, the technology still has a poor prognosis for approximately one-third of women currently seeking assistance for infertility. Understanding the reasons underlying this poor prognosis group of women requires a reflection of the historical evolution of in vitro fertilisation (IVF) from the earliest attempts of the pioneers Robert Edwards and Patrick Steptoe who came together in 1969 [1,2]. Their frustrations with controlled ovarian stimulation led to natural cycle IVF and ignoring the poor prognosis group.

\section{Historical Context}

The corresponding author J.L.Y. has witnessed first-hand the evolution of IVF from 1976 to current day, including periods of working closely with the early IVF pioneers in the embryology arena (Robert Edwards) and the clinical arena (Ian Craft). However, the current "amazing" outcomes from IVF are dependent on protocols that bear little homage to those early workers but are more reflective of those described by the controversial Indian pioneers from Calcutta who achieved their first successful live birth 70 days after the famous 25 July 1978 date of Louise Brown, namely, that of Durga Kanupriya on 3 October 1978. 
The protocol of Subhas Mukherjee, Sunit Mukherjee and SK Bhattacharya [3,4] relied on ovarian stimulation with gonadotropins, trans-vaginal OPU and embryo cryopreservation prior to embryo thawing for a single embryo transfer (SET) as a frozen embryo transfer (FET) procedure in a natural cycle. Now, 43 years later, data reported from the very reliable Australian \& New Zealand Assisted Reproduction Database (ANZARD) show that optimum live birth productivity rates rely on ovarian stimulation with gonadotropins, blastocyst culture and SET procedures, in concert with cryopreservation and subsequent thawing for FETs [5]. In fact, the ANZARD data enable each participating IVF facility to have its verified data accessed via a dedicated website [6]. Our view is that ANZARD provides the most reliable picture for ART outcomes, enabling a clear delineation of the poor prognosis patient, separated out from other limitations within the individual IVF clinics that have a very wide success rating ranging from $9.3 \%$ to $33.2 \%$ live deliveries per initiated treatment cycle [5]. Given that our PIVET facility has success rates in the highest range of the top quartile of the ANZARD report, we believe our studies on the poor prognosis group carry relevance for this commentary.

\section{Factors Limiting the Prognosis}

Apart from the main limiting factor, that of advanced female age $\geq 40$ years, recent studies have shown several other specific variables that can limit the prognosis, namely, the woman's ovarian reserve [7], which itself is attendant upon the antral follicle count (AFC) and the serum anti-Mullerian hormone (AMH) level, as well as the woman's IGF serum profile represented by insulin growth factor-1 (IGF-1), insulin growth factor binding protein-3 (IGFBP-3) and the IGF ratio, being IGFBP-3/IGF-1 [8]. Specific studies conducted at our PIVET facility have excluded variables such as the woman's stature, her body weight, or her body mass index (BMI) as having any relevance to ART treatment outcomes [9], albeit that weight and BMI have a well-known influence on fertility and can be part of the underlying reason for women attending ART facilities. The IVF treatment protocols at PIVET do adjust for BMI variations in selecting the dosage of follicle stimulating hormone (FSH) applied for individual women [10]. Furthermore, following the introduction of intracytoplasmic sperm injection (ICSI) methodology in 1992, most male-factor cases pose no limitation to ART outcomes [11-13]. Even the age of the male partner has minimal influence on outcomes when such studies consider all of the relevant female factors in the analyses [13].

\section{Introducing Adjuvants to Combat Poor-Prognosis Variables}

Given the recently defined confounding variables underlying the poor-prognosis outcomes in current day $[8,9,14,15]$, several adjuvants have been introduced into clinical practice with the view of improving ART outcomes for the poor prognosis group. At PIVET we have been exploring three of these, namely, growth hormone since 2010 [16-20], dehydroepiandrosterone (DHEA) since 2014 [21-23] and melatonin following an encouraging report in 2014 [24,25]. Growth hormone was introduced soon after the favourable report in 2005 on the use of recombinant manufactured growth hormone $(\mathrm{rGH})$ as an adjuvant for older women undergoing assisted reproduction with ICSI [26], with our PIVET team reporting on its use since 2010 [16]. From nearly 50 clinical reports on women with various poor prognosis factors, mostly involving retrospective studies, it appears that embryo utilisation is increased in the vast majority [27], but improvement in live birth rates is reported in under $50 \%$, and those studies all display design weaknesses with problematic confounding variables [27-29]. To minimise the latter, we recently presented data covering a 10-year study period (2011-2020) from our pioneer Australian facility, which involved studies on 3637 autologous IVF \pm ICSI treatment cycles undertaken on 2376 women. This period utilised a stable protocol, where blastocyst culture, single embryo transfers and an advanced cryopreservation program underlies a clinical regimen dictated by a validated FSH-dosing algorithm [30-32]. This unique PIVET algorithm generated $10 \pm 2$ oocytes at ovum pick-up (OPU) for most of the women and rarely $(<4 \%)$ exceeded 20 oocytes; hence, 
three important benefits ensued-firstly, a reduced incidence of ovarian hyperstimulation syndrome (OHSS) to $0.1 \%$; secondly, a reduced need to consider a freeze-all protocol, enabling almost all women to have a fresh embryo transfer; and, thirdly, a pregnancy productivity outcome [33] that is similar for women regardless of their body mass index (BMI), their stature and their ovarian reserve tests across most of the range of AFC and $\mathrm{AMH}$. With respect to the extreme lowest end (category E in the PIVET Algorithm with AFC $<5$ antral follicles and $\mathrm{AMH}<5 \mathrm{pmol} / \mathrm{L}$ ), this aspect is difficult to separate out from the consideration of advanced age, as the algorithm defines a requirement for maximal FSH dosing; hence, oocyte numbers per OPU tend to be optimised, albeit at numbers under $10 \pm 2$. Two large studies examining three adjuvants have recently been reported from PIVET, the first analysing the influence of $\mathrm{rGH} \pm$ melatonin [25] and the second from the same period reporting on $\mathrm{rGH} \pm$ DHEA [23].

\section{Poor Ovarian Responder vs. Poor-Prognosis Criteria}

PIVET studies on the poor prognosis group of women need to be considered as distinct from those applying the Bologna criteria for the poor ovarian responder (POR). The latter is focused on the retrieval of few oocytes at OPU as a reflection of the woman's age and her ovarian reserve tests (AFC and AMH) [34]. However, PIVET has not focused on oocyte numbers at OPU, rather reporting on embryo utilisation rates (those oocytes that fertilise and then contribute as FETs or are cryopreserved for potential future FETs). PIVET studies on POR (Bologna) are in agreement with most reports and tend not to show any benefits from $\mathrm{rGH}$, DHEA or Melatonin adjuvants, with respect to neither oocyte numbers nor live birth rates (from neither fresh embryo SETs nor the productivity of all fresh and frozen ETs). In fact, the live birth productivity rates are significantly higher for those women not utilising adjuvants. However, if one factors in the consideration of embryo quality, a different picture emerges. We regard a standard favourable IVF \pm ICSI cycle as one generating $10 \pm 2$ oocytes leading to one fresh SET procedure and 1-3 blastocysts with Gardner grading of 3BB or higher [35], cryopreserved by Vitrification applying the Kuwayama technique [36]. Across the age ranges, the mean number of oocytes is 11.1 for women $<35$ years, 9.4 for women 35-39 years, reducing to 6.5 for women aged 40-44 years and 4.0 for those women aged $\geq 45$ years. Our two most recent studies classify those treatment cycles where, after the fresh SET, no embryos proved suitable for cryopreservation, as one of poor embryo quality, affecting almost $47.5 \%$ of the women having autologous treatments at PIVET.

\section{Key Laboratory and Clinical Outcomes from Adjuvant Studies}

One or more of the three adjuvants was utilised in $28 \%$ of autologous IVF \pm ICSI cycles conducted at PIVET. Oocyte numbers at OPU were not influenced by any of the adjuvants, nor was the oocyte fertilisation rate. However, for those women classified as acceptable embryo quality (with at least one embryo cryopreserved), embryo utilisation was higher from rGH for those aged $>40$ years. However, our studies did not translate into any improvement in the live birth productivity rates, which were always significantly in favour of the women using no adjuvants. Neither melatonin nor DHEA (alone or combined) showed any benefit on embryo utilisation rates, nor on live birth rates in this acceptable embryo quality category. However, focusing on those women with poor embryo quality, both rGH and DHEA (alone or combined) did show significantly elevated embryo utilisation rates. These elevated rates were especially marked among those women aged $\geq 40$ years with low ovarian reserve indices as well as those with low IGF-1 levels or those with repetitive implantation failure RIF (classified as no pregnancy ensuing from $\geq 3$ embryo transfer procedures). With respect to live births, all the poor embryo quality subgroups showed low rates, including those who did not utilise adjuvants, and there were no significant differences among the rates for nil vs. either rGH or DHEA or rGH+DHEA. Melatonin showed no benefits for neither embryo utilisation nor for live birth rates, and the rates were significantly lower than other adjuvants or for nil adjuvants. With further studies investigating the influence of several poor prognosis factors, we did show that 
those women with poor embryo quality and with advanced age $\geq 40$ years, along with AFC Group E and low IGF-1, had significantly higher embryo utilisation rates, which translated through to elevated pregnancies, reduced miscarriages (early pregnancy losses) and significantly higher live birth outcomes [20].

\section{Validity of Data}

Critics of adjuvant use would say "I told you so", meaning adjuvants are not useful $[37,38]$ and add to the expense of an already costly treatment mode for infertility, especially that of rGH. However, we would point out that the vast number of studies reported have been conducted on small numbers, far fewer than the 400 required for a relevant randomised controlled trial. Although our studies are observational, with retrospective analysis, they are conducted on large numbers embracing 3637 cycles overall and include 1727 cycles classified as poor prognosis due to poor embryo quality. Furthermore, the treatment protocols are totally standardised with ovarian stimulation conducted under a strictly controlled FSH-dosing algorithm designed to totally avoid the problem of OHSS (which it did), as well as avoiding the disappointment of a freeze-all strategy, which occurred infrequently as the rate of women with $\geq 20$ oocytes was only $4 \%$, indicating that the algorithm is working effectively at both ends of the spectrum (providing adequate oocytes across a rising age range and avoiding excessive responses for those with high ovarian reserve tests). Furthermore, the SET policy and single embryo FET protocol provide the optimum live birth productivity rate from a single initiated autologous IVF \pm ICSI treatment cycle. This approach also enables a continuing satisfactory live birth rate for RIF cases [39].

\section{Potential Benefits from rGH and Possibly from DHEA}

Laboratory studies do generally support the role of rGH in improving oocyte quality [18,40-43], and there is plausible evidence for the consideration of adult growth hormone deficiency (AGHD) underlying some cases of infertility, particularly where other pituitary dysfunction is present $[19,44-49]$. However, we have shown that only $12 \%$ of infertile women will demonstrate normal serum $\mathrm{GH}$ levels $(\geq 3.0 \mathrm{ng} / \mathrm{mL})$ on a morning blood test, hence the need to apply an indirect marker, such as the IGF profile [14]. Given the clear association of IVF prognosis with the IGF profile and particularly with IGF-1 levels [15], the finding that rGH can significantly elevate and normalise low levels over a 6-week treatment course [20] implies that increased live birth rates could be expected. Perhaps the failure of the numerous reported studies on short trials of rGH treatment means that the hormone should be given for a longer period. In this context, we would encourage research studies involving a deeper evaluation of women categorised as having a poor prognosis in IVF. Such should include the gold-standard insulin stimulation test or the less risky glucagon stimulation test, which would define the presence of AGHD. We recommend that prolonged $\mathrm{rGH}$ treatment over 12 weeks or more should be investigated in clearly defined trials. Similarly, DHEA treatment could be evaluated in those women with a defined hormonal deficiency.

\section{Summary of Adjuvant Studies from PIVET}

The three adjuvants under consideration for this commentary namely, melatonin, $\mathrm{rGH}$ and DHEA, have been extensively studied at PIVET and reported sequentially from 2010. The findings can be summarised as follows:

1. All of the data generated on melatonin use indicate clear evidence for neither embryological nor clinical benefit. Furthermore, we could not detect any suggestion of a clinical group comprising poor prognostic factors that might be useful to explore in defined research trials.

2. The trials on rGH use provided two clear messages. Firstly, where IVF treatments have resulted in at least one supernumerary embryo (after a fresh SET) reaching blastocyst cryopreservation, such cases will not show any benefit in embryo utilisation or in live birth productivity rates. In fact, the data strongly imply that live birth outcomes 
may be significantly reduced. Secondly, there are highly suggestive data that women classified as having poor embryo quality (nil embryos cryopreserved) will benefit from $\mathrm{rGH}$ use if they also have a very low ovarian reserve (AFC $<5$ follicles $\pm \mathrm{AMH}$ $<5 \mathrm{pmol} / \mathrm{L}$ ). Furthermore, the data for $\mathrm{rGH}$ benefit are stronger where additional poor prognosis factors can be shown, such as advanced age ( $\geq 40$ years), a low IGF profile (e.g., IGF-1 in the lowest quartile) or RIF ( $\geq 3$ failed ETs).

3. With respect to DHEA, the data are in accordance with those for $\mathrm{rGH}$, albeit with benefits not as pronounced according to levels of significance. Firstly, for women with at least one embryo cryopreserved after a SET procedure, there is no improvement in embryo utilisation or in clinical livebirth productivity rates; in fact, there are data suggesting a reduced pregnancy and live birth outcome. Secondly, there are suggestive data that for women classified as having poor embryo quality, embryo utilisation is significantly improved with DHEA alone and more strongly with the combination of DHEA with rGH. Live birth productivity is similarly enhanced for those women with additional poor prognosis factors, being significantly stronger with the combination of DHEA with $\mathrm{rGH}$, albeit that $\mathrm{rGH}$ alone shows the highest levels of significance across the grouped studies.

\section{Conclusions}

In view of widespread scepticism concerning the use of around 50 adjuvants or addons in IVF, we believe the observational evidence from matched retrospective studies concerning three of them enables the conclusion that melatonin has no relevant utility, but rGH and likely DHEA have highly suggestive benefits warranting appropriate study. Such studies should be focused on those women who fail to generate any embryos suitable for cryopreservation following SET and also display a very low ovarian reserve as well as one or more poor prognosis factors in addition to the finding of poor embryo quality. Those poor prognosis factors should include the possibility of AGHD, which should be investigated. Furthermore, future trials should consider the need to extend rGH treatment across a period of at least 12 weeks (rather than PIVET's 6-week trials) to cover for the maturational period of activated oocytes. In view of the similarly suggestive findings of a clinical benefit from DHEA, such studies should be conducted in concert, as there would be major cost benefits, as well as long-term clinical implications, if it can be shown that DHEA can match the rGH benefits.

Author Contributions: The studies from PIVET were conceived by Medical Director J.L.Y., who established the database at PIVET Medical Centre with the assistance of IT Consultant and Data Manager P.M.H., who is co-author of this commentary. J.L.Y. has led six consultant clinicians at PIVET, establishing clinical protocols with patient information sheets enabling appropriately informed consenting arrangements for the use of adjuvants in IVF. Three of the clinicians have sub-specialty qualifications in reproductive medicine, including andrology and reproductive endocrinology. The authors are grateful for the clinicians who were involved in recruiting patients and counselling them with respect to the use of adjuvants. All authors assisted in the data analyses as well as in the preparation of the tables and figures for the published reports. The manuscript was written by J.L.Y., and P.M.H. has read and agreed to its content. All authors have read and agreed to the published version of the manuscript.

Funding: This research received no external funding.

Institutional Review Board Statement: Reporting of the data presented here was approved under Curtin University Human Ethics Committee approval no. RD-25-10, general approval for retrospective data analysis in 2010, updated in 2015 and again further updated recently in August 2020.

Informed Consent Statement: PIVET is accredited with both the self-regulatory National Australian Reproductive Technology Committee (RTAC) as well as the Reproductive Technology Council (RTC) of Western Australia. Consent forms received approval under both regulatory bodies. The assay laboratory is accredited on an annual basis by the National Australian Testing Authority (NATA). 
Data Availability Statement: The data presented in this study are available on request from the corresponding author. The data are not publicly available due to privacy reasons (Private Clinic comprising private patients).

Acknowledgments: We are grateful for the close working relationship between PIVET®Medical Centre and CLINIPATH®Pathology, which carried out the assays involved in the IGF profile and is also accredited by NATA. The nursing team at PIVET was fastidious in detailing clinical outcomes into registers and then into the Filemaker database. Alison Pusey was especially successful in tracking the outcome of each pregnancy, many of which resulted in deliveries in regional locations and sometimes overseas.

Conflicts of Interest: The entire project was funded internally at PIVET without any external or commercial contributions. The authors declare no conflict of interest.

\section{References}

1. Yovich, J.L.; Craft, I.L. Founding pioneers of IVF: Independent innovative researchers generating livebirths within 4 years of the first birth. Reprod. Biol. 2018, 18, 317-323. [CrossRef] [PubMed]

2. Yovich, J.L. Founding pioneers of IVF Update: Independent innovative researchers generating livebirths within 4 years of the first birth. Reprod. Biol. 2020, 20, 111-113.

3. Mukherjee, S.; Mukherjee, S.; Bhattacharya, S.K. The feasibility of long term cryogenic freezing of viable human embryos-A brief pilot study report. Indian J. Cryog. 1978, 3, 80 .

4. Sharma, R.S.; Saxena, R.; Singh, R. Infertility \& assisted reproduction: A historical \& modern scientific perspective. Indian J. Med. Res. 2018, 148, 10-14.

5. Newman, J.E.; Paul, R.C.; Chambers, G.M. Assisted Reproductive Technology in Australia and New Zealand 2018; National Perinatal Epidemiology and Statistics Unit, the University of New South Wales: Sydney, Australia, 2020; pp. 1-83.

6. Your IVF Success Estimator. Find an Australian IVF Clinic. Australian E New Zealand Assisted Reproduction Database; University of New South Wales: Sydney, Australia, 2020. Available online: https:/ /www.yourivfsuccess.com.au (accessed on 3 March 2021).

7. Keane, K.; Cruzat, V.F.; Wagle, S.; Chaudhary, N.; Newsholme, P.; Yovich, J. Specific ranges of anti-Mullerian hormone and antral follicle count correlate to provide a prognostic indicator for IVF outcome. Reprod. Biol. 2017, 17, 51-59. [CrossRef] [PubMed]

8. Yovich, J.L.; Zaidi, S.; Nguyen, M.D.K.; Hinchliffe, P.M. Measuring IGF-1 and IGFBP-3 profiles in women seeking assisted reproduction; relationship to ovarian reserve parameters (Study 2). GSC Biol. Pharm. Sci. 2020, 13, 35-53. [CrossRef]

9. Yovich, J.L.; Zaidi, S.; Nguyen, M.D.K.; Hinchliffe, P.M. Measuring IGF-1 and IGFBP-3 profiles in women seeking assisted reproduction; relationship to clinical parameters (Study 1). J. Pers. Med. 2020, 10, 122. [CrossRef]

10. Yovich, J.L. How to Prepare the Egg and Embryo to Maximise IVF Success. In Monitoring the Stimulated IVF Cycle. Section II: Stimulation for IVF; Kovacs, G.K., Rutherford, A.J., Gardner, D.K., Eds.; Cambridge University Press: Cambridge, UK, 2019; pp. 94-120.

11. Yovich, J.L.; Conceicao, J.L.; Marjanovich, N.; Ye, Y.; Hinchliffe, P.M.; Dhaliwal, S.S.; Keane, K.N. An ICSI rate of $90 \%$ minimizes complete failed fertilization and provides satisfactory implantation rates without elevating fetal abnormalities. Reprod. Biol. 2018, 18, 301-311. [CrossRef] [PubMed]

12. Yovich, J.L.; Conceicao, J.L.; Wong, J.; Marjanovic, N.; Wicks, R.; Hinchliffe, P.M. Fertlization by ICSI generates a higher number of live births than IVF in a pioneer facility applying $>90 \%$ single blastocyst-stage embryo transfers. GSC Biol. Pharm. Sci. 2021, 15, 87-103. [CrossRef]

13. Mariappen, U.; Keane, K.N.; Hinchliffe, P.M.; Dhaliwal, S.S.; Yovich, J.L. Neither male age nor semen parameters influence clinical pregnancy or live birth outcomes from IVF. Reprod. Biol. 2018, 18, 324-329. [CrossRef] [PubMed]

14. Yovich, J.L.; Zaidi, S.; Nguyen, M.D.K.; Hinchliffe, P.M. Measuring IGF-1 and IGFBP-3 profiles in women seeking assisted reproduction; relationship to serum growth hormone levels (Study 3). GSC Biol. Pharm. Sci. 2020, 13, 32-53. [CrossRef]

15. Yovich, J.L.; Zaidi, S.; Nguyen, M.D.K.; Hinchliffe, P.M. Measuring IGF-1 and IGFBP-3 profiles in women seeking assisted reproduction; relevance to clinical outcomes from in vitro fertilization (Study 5). GSC Biol. Pharm. Sci. 2020, 13, 79-96. [CrossRef]

16. Yovich, J.L.; Stanger, J.D. Growth hormone supplementation improves implantation and pregnancy productivity rates for poor-prognosis patients undertaking IVF. Reprod. Biomed. Online 2010, 21, 37-49. [CrossRef]

17. Keane, K.N.; Yovich, J.L.; Hamidi, A.; Hinchliffe, P.M.; Dhaliwal, S.S. Single-centre retrospective analysis of growth hormone supplementation in IVF patients classified as poor-prognosis. BMJ Open 2017, 7, e018107. [CrossRef]

18. Keane, K.N.; Ye, Y.; Regan, S.L.P.; Dhaliwal, S.S.; Yovich, J.L. Live birth outcomes of vitrified embryos generated under growth hormone stimulation are improved for women categorized as poor-prognosis. Clin. Exp. Reprod. Med. 2019, 46, 178-188. [CrossRef]

19. Yovich, J.L.; Ye, Y.; Regan, S.L.P.; Keane, K.N. The evolving concept of poor-prognosis for women undertaking IVF and the notion of growth hormone as an adjuvant; a single-center viewpoint. Front. Endocrinol. 2019, 10, 808. [CrossRef] [PubMed]

20. Yovich, J.L.; Zaidi, S.; Nguyen, M.D.K.; Hinchliffe, P.M. Measuring IGF-1 and IGFBP-3 profiles in women seeking assisted reproduction; response of women categorised as poor prognosis to growth hormone adjuvant therapy (Study 4). GSC Biol. Pharm. Sci. 2020, 13, 64-78. [CrossRef] 
21. Keane, K.N.; Hinchliffe, P.M.; Namdar, N.; Conceicao, J.L.; Newsholme, P.; Yovich, J.L. Novel dehydroepiandrosterone troche supplementation improves the serum androgen profile of women undergoing in vitro fertilization. Drug Des. Dev. Ther. 2015, 9, 5569-5578. [CrossRef]

22. Keane, K.N.; Hinchliffe, P.M.; Rowlands, P.K.; Borude, G.; Srinivasan, S.; Dhaliwal, S.S.; Yovich, J.L. DHEA Supplementation confers no additional benefit to that of growth hormone on pregnancy and live birth rates in IVF patients categorized as poor prognosis. Front. Endocrinol. 2018, 9, 14. [CrossRef] [PubMed]

23. Yovich, J.L.; Srinivasan, S.; Sillender, M.; Gaur, S.; Rowlands, P.; Hinchliffe, P.M. Applying growth hormone as an adjuvant to correct poor prognosis outcomes in IVF: Study 2 compares dehydroepiandrosterone. GSC Biol. Pharm. Sci. 2021, 16, 164-190. [CrossRef]

24. Fernando, S.; Osianlis, T.; Vollenhoven, B.; Wallace, E.; Rombauts, L. A pilot double-blind randomised placebo-controlled doseresponse trial asssessing the effects of melatonin on infertility treatment (MIART): Study protocol. BMJ Open 2014, 4, e0059866. [CrossRef]

25. Yovich, J.L.; Srinivasan, S.; Sillender, M.; Gaur, S.; Rowlands, P.; Hinchliffe, P.M. Applying growth hormone as an adjuvant to correct poor prognosis outcomes in IVF: Study 1 compares melatonin. GSC Biol. Pharm. Sci. 2021, 16, 219-238. [CrossRef]

26. Tesarik, J.; Hazout, A.; Mendoza, C. Improvement of delivery and live birth rates after ICSI in women aged $>40$ years by ovarian co-stimulation with growth hormone. Hum. Reprod. 2005, 20, 2536-2541. [CrossRef] [PubMed]

27. Yovich, J.L.; Regan, S.L.; Zaidi, S.N.; Keane, K.N. The concept of growth hormone deficiency affecting clinical prognosis in IVF. Front. Endocrinol. 2019, 10, 650. [CrossRef]

28. Yovich, J.L.; Ye, Y.; Keane, K.N. Growth hormone adjuvant trial for poor responders undergoing IVF. Eur. J. Obstet. Gynecol. 2019, 236, 249-251. [CrossRef] [PubMed]

29. Bortoletto, P.; Spandorfer, S. Growth hormone: In search of the holy grail for poor responders (or a felony). Fertil. Steril. 2020, 114, 63-64. [CrossRef] [PubMed]

30. Yovich, J.; Stanger, J.; Hinchliffe, P. Targeted gonadotrophin stimulation using the PIVET algorithm markedly reduces the risk of OHSS. Reprod. Biomed. Online 2012, 24, 281-292. [CrossRef] [PubMed]

31. Yovich, J.L.; Alsbjerg, B.; Conceicao, J.L.; Hinchliffe, P.M.; Keane, K.N. PIVET rFSH dosing algorithms for individualized controlled ovarian stimulation enables optimized pregnancy productivity rates and avoidance of ovarian hyperstimulation syndrome. Drug Des. Dev. Ther. 2016, 10, 2561-2573. [CrossRef]

32. Yovich, J.L.; Hinchliffe, P.M.; Lingam, S.; Srinivasan, S.; Keane, K.N. Adjusting the PIVET rFSH dosing algorithm for the biosimilar Bemfola product. JFIV Reprod. Med. Genet. Stem Cell Biol. 2018, 5, 1-4.

33. Yovich, J.L.; Stanger, J.D.; Keane, K.N. Cumulative live birth rate: An outmoded term. JFIV Reprod Med Genet 2016, 4, 165. [CrossRef]

34. Ferraretti, A.P.; La Marca, A.; Fauser, B.C.; Tarlatzis, B.; Nargund, G.; Gianaroli, L.; ESHRE Working Group on Poor Ovarian Response Definition. ESHRE consensus on the definition of 'poor response' to ovarian stimulation for in vitro fertilization: The Bologna criteria. Hum. Reprod. 2011, 26, 1616-1624. [CrossRef] [PubMed]

35. Yovich, J.L.; Conceicao, J.; Hinchliffe, P.; Keane, K. Which blastocysts should be considered for genetic screening? Hum. Reprod. 2015, 30, 1743-1745. [CrossRef] [PubMed]

36. Kuwayama, M.; Vajta, G.; Kato, O.; Leibo, S.P. Highly efficient vitrification method for cryopreservation of human oocytes. Reprod. Biomed. Online 2005, 11, 300-308. [CrossRef]

37. Farquhar, C. Add-ons for assisted reproductive technology: Can we be honest here? Fertil. Steril. 2019, 112, 971-972. [CrossRef]

38. Norman, R.J.; Alvino, H.; Hull, L.M.; Mol, B.W.; Hart, R.J.; Kelly, T.-L.; Rombauts, L. Human growth hormone for poor responders: A randomized placebo-controlled trial provides no evidence for improved live birth rate. Reprod. Biomed. Online 2019, 38, 908-915. [CrossRef] [PubMed]

39. Mustafa, K.B.; Keane, K.N.; Walz, N.L.; Mitrovic, K.I.; Hinchliffe, P.M.; Yovich, J.L. Live Birth Rates are satisfactory following multiple IVF treatment cycles in poor prognosis patients. Reprod. Biol. 2017, 17, 34-41. [CrossRef] [PubMed]

40. Weall, B.M.; Al-Samerria, S.; Conceicao, J.; Yovich, J.L.; Almahbobi, G. A direct action for GH in improvement of oocyte quality in poor-responder patients. Reproduction 2015, 149, 147-154. [CrossRef]

41. Regan, S.L.P.; Knight, P.G.; Yovich, J.L.; Arfuso, F.; Dharmarajan, A. Growth hormone during in vitro fertilization in older women modulates the density of receptors in granulosa cells, with improved pregnancy outcomes. Fertil. Steril. 2018, 110, 1298-1310. [CrossRef]

42. Tesarik, J.; Galán-Lázaro, M.; Conde-López, C.; Chiara-Rapisarda, A.M.; Mendoza-Tesarik, R. The effect of rGH administration on oocyte and zygote quality in young women with repeated implantation failure after IVF. Front. Endocrinol. 2020, 11, 519-572. [CrossRef]

43. Devesa, J.; Caicedo, D. The role of growth hormone on ovarian functioning and ovarian angiogenesis. Front. Endocrinol. 2019, 10, 450. [CrossRef]

44. Ipsa, E.; Cruzat, V.F.; Kagize, J.N.; Yovich, J.L.; Keane, K.N. Growth Hormone and Insulin-like growth factor in reproductive tissues. Front. Endocrinol. 2019, 10, 777. [CrossRef]

45. Yovich, J.L.; Srinivasan, S.; Sillender, M.; Gaur, S.; Rowlands, P.; Hinchliffe, P.M. Using growth hormone as an adjuvant in IVF: Live birth outcomes from various poor prognosis scenarios. GSC Biol. Pharm. Sci. 2021, 15, 063-080. [CrossRef] 
46. Li, J.; Chen, Q.; Wang, J.; Huang, G.; Ye, H. Does growth hormone supplementation improve oocyte competence and IVF outcomes in patients with poor embryonic development? A randomized controlled trial. BMC Pregnancy Childbirth 2020, 20, 1-10. [CrossRef] [PubMed]

47. Zhang, Y.; Zhang, C.; Shu, J.; Guo, J.; Chang, H.-M.; Leung, P.C.K.; Sheng, J.-Z.; Huang, H. Adjuvant treatment strategies in ovarian stimulation for poor responders undergoing IVF: A systematic review and network meta-analysis. Hum. Reprod. Update 2020, 26, 247-263. [CrossRef] [PubMed]

48. Yang, P.; Wu, R.; Zhang, H. The effect of growth hormone supplementation in poor ovarian responders undergoing IVF or ICSI: A meta-analysis of randomized controlled trials. Reprod. Biol. Endocrinol. 2020, 18, 76. [CrossRef] [PubMed]

49. Tesarik, J.; Yovich, J.L.; Menezo, Y. Editorial: Growth hormone in Fertility and infertility: Physiology, pathology, diagnosis and treatment. Front. Endocrinol. 2021, 12, 621722. [CrossRef] [PubMed] 